\title{
A Review of Some Monte Carlo Simulation Methods for Turbulent Systems
}

\author{
Peter R. Kramer \\ Department of Mathematical Sciences \\ Rensselaer Polytechnic Institute, Troy, NY 12180
}

October 2, 2000

\section{Abstract}

We provide a brief overview of some Monte Carlo methods which have been used to simulate systems with a turbulent fluid component. We discuss two main classes of simulation approaches: an "Eulerian" class which is based on a random velocity field model defined on a fixed coordinate system, and a "Lagrangian" class in which the motion of fluid particles and immersed particles are instead stochastically modeled. The main aim of this article is to expose these novel simulation approaches to turbulent and complex fluid systems to a broader readership familiar with stochastic processes and to provide some pointers to the literature.

\section{Introduction}

A continuing challenge is the numerical simulation of turbulent systems, in which the fluid is forced sufficiently vigorously so as to generate a disordered and unpredictable structure over a wide range of scales $[47,74]$. The key difficulty is the inability for even large computers to resolve all of the active scales in systems with strong turbulence in a direct numerical simulation based on the fundamental Navier-Stokes equations [43, 54]. A numerical representation of turbulence, however, is crucial in computer simulation studies in many applied fields, ranging from atmosphere-ocean dynamics (including weather and climate prediction) [11, 43, 47], combustion [7, 33, 45, 62, 80, 85], turbulent diffusion [48], mixing processes and numerous other engineering situations [36, 54]. These applications do not require a turbulence simulation with full fidelity - only certain key features of the turbulence need to be represented. On the other hand, models which account for turbulence only through an enhanced diffusivity coefficient are often insufficient $[43,54,77]$. This is particularly the case in reaction and mixing processes where the fine-scale fluctuations in the immersed chemical species play an important role, and these are completely ignored in a crude eddy diffusion model and not well represented in large eddy simulations [33, 45].

Monte Carlo approaches are an attractive option for turbulence simulations due both to their capacity for investigating systems with many degrees of freedom and to their natural generation of a disordered velocity field structure and irregular particle trajectories. Indeed, it is difficult to conceive of a deterministic mechanism for generating a velocity field with disordered fluctuations over a wide range of scales, other than by an expensive direct numerical simulation which resolves all those scales! Most recent methods which have been used to simulate turbulent systems can be classified into two categories. In the first, which we call the "Eulerian" fluid simulation approach, a velocity field is generated over a prescribed spatial domain, but by a direct stochastic construction 
rather than the much more expensive simulation of the nonlinear Navier-Stokes PDE's. That is, the turbulent velocity field $\mathbf{u}(\mathbf{x}, t)$ is replaced by a stochastic model velocity field $\mathbf{u}_{\text {mod }}(\mathbf{x}, t)$, which is intended to mimic the important features of turbulence desired for the application. The trajectory $\mathbf{X}(t)$ of any immersed particle representing, for example, a tracer, pollutant, or chemical reactant, is then computed using the local value of the stochastically constructed velocity field $\mathbf{u}_{\bmod }(\mathbf{X}(t), t)$. For example, a passive, light tracer particle's motion is often modelled by the stochastic differential equation [48]:

$$
d \mathbf{X}(t)=\mathbf{u}(\mathbf{X}(t), t) d t+\sqrt{2 \kappa} d \mathbf{W}(t),
$$

where a Brownian motion component $\sqrt{2 \kappa} d \mathbf{W}(t)$ has been added to simulate the effects of molecular diffusivity $\kappa$ [48]. Multiple immersed particles are simulated by similar stochastic equations, evaluated using the same realization of the turbulent velocity field $\mathbf{u}$ but independent Brownian motions. The influence of particle buoyancy, inertia, drag, and memory effects resulting from interaction with the dynamical fluid can be incorporated in more complete and complex equations $[53,69,70,76]$; see also Subsection 4.2.

The main challenge with the Eulerian fluid simulation approach is the efficient generation of the model synthetic velocity field $\mathbf{u}_{\text {mod }}(\mathbf{x}, t)$, particularly when one wishes to mimic well the scaling properties of turbulent flows. Once the synthetic velocity field is simulated, the integration of the particle trajectories, particularly for passive tracer particles (1), can be handled by a standard techniques $[15,17,23,28,88]$. For some special velocity field models, such as rapidly decorrelating flows $[37,39,48]$ and shear flows $[3,4,32]$, it is possible to simulate directly (and rigorously) the statistics of the velocity $\mathbf{u}(\mathbf{X}(t), t)$ observed by an immersed particle or collection of particles without having to simulate the random velocity field $\mathbf{u}(\mathbf{x}, t)$ over a full spatial domain $[23,24,26$, 81]. The possibility of such a shortcut is, however, limited to special models. We discuss some suitable Monte Carlo methods for generating synthetic turbulent velocity field models in Section 3.

An alternative "purely Lagrangian" simulation approach bases itself not on the construction of a stochastic velocity field model but on a stochastic model for the evolution of particles in the system. In many applications of the purely Lagrangian approach, there is never any reference to a fluid velocity field; the effects of turbulence are already modeled by the equations of evolution of the immersed particles. The purely Lagrangian approach has however also been used to simulate the motion of the turbulent fluid itself $[60,84]$ by representing the fluid as a collection of particles which evolve according to some stochastic equations. The fluid properties at a given position and time are then defined in terms of the averaged properties of fluid particles in the vicinity.

The evolution equations for the position $\mathbf{X}(t)$ of a particle in a purely Lagrangian Monte Carlo simulation is typically based on a continuous Markov evolution for the particle velocity and position:

$$
\begin{aligned}
& d \mathbf{X}(t)=\mathbf{V}(t) d t \\
& d \mathbf{V}(t)=\mathbf{A}(\mathbf{X}(t), \mathbf{V}(t)) d t+\mathcal{B}(\mathbf{X}(t), \mathbf{V}(t)) d \mathbf{W}(t) .
\end{aligned}
$$

The (deterministic) coefficients $\mathbf{A}(\mathbf{x}, \mathbf{v})$ and $\mathcal{B}(\mathbf{x}, \mathbf{v})$ are defined by the particular stochastic model, and there is much ongoing research as to both theoretically desirable $[41,71,72,78]$ and practically desirable [86] forms for these coefficients. In chemically reacting or combusting systems, the equations (2) are augmented by another stochastic equation describing the local chemical composition at the particle's location [33,62,67]. The simulated particles in such applications should not be viewed as physical particles but rather as markers keeping track of the effects of fluid convection.

The literature on developments and application of the purely Lagrangian simulation approach is proliferating, and in the introductory spirit of this paper, we confine ourselves to describing some foundational ideas in Section 4. We remark here that the Probability Density Function (PDF) and related Filtered Mass Density Function (FMDF) methodology developed by Pope and coworkers $[64,67,68,80,86]$ and applied by several groups to problems in combustion $[33,62]$ is a 
notable case of a purely Lagrangian simulation approach. We also would like to draw the reader's attention to the burgeoning field of Dissipative Particle Dynamics (DPD) [1, 19, 20, 21, 27, 30, 63], in which the small-scale dynamics of complex fluids, such as polymeric liquids, are simulated by a purely Lagrangian approach similar of similar character to those used in turbulence studies. While these fluids are not typically turbulent, the simulation models entail a stochastic component due to thermal fluctuations which play an important role on the resolved microscales of motion.

Some novel Monte Carlo approaches have been proposed and developed which do not fall cleanly into one of the two categories described above. For example, Kurbanmuradov and Sabelfeld [41, 72] have formulated a hybrid Eulerian-Lagrangian fluid simulation algorithm for particle dispersion which endeavors to draw from the strengths of each approach. Kerstein [35] has over the last decade developed a stochastic model of turbulent mixing which can be simulated on a fixed line segment representing a linear cut of the turbulent flow. The one-dimensionality of the model permits all scales to be resolved, so that fine-scale features of immersed particle and chemical mixing can be simulated. This one-dimensional turbulence model has been coupled to models for stratus cloud-top entrainment instability [40] and chemical reaction [34, 56], where the outcome is sensitive to small-scale fluctuations in the density of entrained air and reactants, respectively. In another direction, Biechele, Breuer, and Petruccione $[6,8]$ have developed a Monte Carlo method for simulating the deterministic evolution of decaying turbulence.

We close this brief introductory review in Section 5 with some general remarks about the comparative utility of the Eulerian fluid and purely Lagrangian simulation approaches for turbulent systems.

\section{Eulerian Fluid Simulation Methods}

As mentioned in the introduction, the primary challenge in most Eulerian fluid Monte Carlo simulations is the generation of a synthetic random velocity field $\mathbf{u}_{\bmod }(\mathbf{x}, t)$ which has certain statistics and features mimicing those of a turbulent fluid in the regime of interest. For example, a fully developed turbulent flow at sufficiently high Reynolds number should possess a wide inertial range of scales over which the statistics of the velocity field assume a self-similar fractal structure $[10,25,43,75]$. A quantitative way to partially express this criterion is

$$
\left\langle|\mathbf{u}(\mathbf{x}+\mathbf{r}, t)-\mathbf{u}(\mathbf{x}, t)|^{2}\right\rangle=S_{v}^{I}|\mathbf{r}|^{2 H} \text { for } L_{K} \ll|\mathbf{r}| \ll L_{0}
$$

where $\langle\cdot\rangle$ denotes a statistical average, $0<H<1$ is the Hurst exponent [49] which takes the Kolmogorov value $H=1 / 3$ for fully developed turbulence, $L_{K}$ is the dissipation length scale and $L_{0}$ is the integral length scale which define the extent of the inertial scaling range, and $S_{v}^{I}$ is a (dimensional) scaling prefactor. Other desiderata in turbulence simulations are incompressibility of the fluid and appropriate geometric symmetries such as isotropy.

To focus on main ideas and minimize notational complexity in this brief review, we will describe four techniques for constructing one-dimensional, homogenous, steady, mean zero Gaussian random fields $u(x)$ with spatial structure prescribed equivalently through its correlation function $R(x)$ or energy spectrum $E(k)[77,87]$ :

$$
R(x)=\left\langle u\left(x^{\prime}\right) u\left(x^{\prime}+x\right)\right\rangle=\int_{-\infty}^{\infty} e^{2 \pi i k x} E(|k|) d k .
$$

After formulating the main ideas behind four simulation techniques, we briefly discuss some findings from applications of these techniques on some test problems $[15,18,32]$ and offer some guidelines for their use. Our presentation must be brief here; more comprehensive reviews may be found in [31] and [48, Sec. 6]. 
All four methods which we will describe can be carried over to multi-dimensional and incompressible flows through straightforward extension [73] or random plane wave construction [16, 46]. Evolving Gaussian Markov flows $u(x, t)$ built from Ornstein-Uhlenbeck processes can also be generated by adaptation of the techniques presented $[9,72]$. Much more challenging, however, is the simulation of non-Gaussian random velocity fields with prescribed statistics, as would be needed for a faithful representation of the effects of small-scale turbulent intermittency [74, 75]. We emphasize, however, that much has been and can still be learned from exploration of turbulent diffusion and combustion in simplified Gaussian models with various spatio-temporal structures, particularly those with self-similar scaling regimes [48].

\subsection{Fourier Method}

The Fourier method, utilized in $[82,83]$, is based on the spectral representation formula for a homogenous Gaussian random field [87, Sec. 8], which can be expressed in terms of real-valued functions as

$$
u(x)=\int_{0}^{\infty} \cos (2 \pi k x) E^{1 / 2}(k) d W_{1}(k)+\int_{0}^{\infty} \sin (2 \pi k x) E^{1 / 2}(k) d W_{2}(k) .
$$

Here $d W_{1}(k)$ and $d W_{2}(k)$ are independent real-valued Gaussian white noise measures [87, Sec. 8] with the formal properties

$$
\begin{gathered}
\left\langle d W_{1}(k)\right\rangle=\left\langle d W_{2}(k)\right\rangle=0, \\
\left\langle d W_{1}(k) d W_{1}\left(k^{\prime}\right)\right\rangle=\left\langle d W_{2}(k) d W_{2}\left(k^{\prime}\right)\right\rangle==\delta\left(k-k^{\prime}\right) d k d k^{\prime} .
\end{gathered}
$$

In the standard implementation of the Fourier method, the stochastic Fourier integral (4) is truncated and discretized by an equispaced Riemann sum approximation:

$$
u_{\text {Four }}(x)=\sum_{j=0}^{M} E^{1 / 2}(|j| \Delta k) \sqrt{\Delta k}\left[\xi_{j} \cos 2 \pi j \Delta k x+\eta_{j} \sin 2 \pi j \Delta k x\right],
$$

where $\Delta k$ is the wavenumber spacing and $M$ denotes the highest Fourier mode retained. The $\left\{\xi_{j}\right\}_{j=0}^{M}$ and $\left\{\eta_{j}\right\}_{j=0}^{M}$ are independent standard (real-valued) Gaussian random variables, meaning that each has mean zero and unit variance.

We note that the Fourier method generates a velocity field $u_{\text {Four }}(x)$ with an inherent spatial period $(\Delta k)^{-1}$. This feature can create often undesirable artifacts in turbulent diffusion simulations, particularly over long times [14]. One way to reduce the effect of the periodicity is to use a nonequally spaced discretization of the stochastic Fourier integral, but the choice of how to do this would be ad hoc.

\subsection{Randomization Method}

A more attractive simulation technique based on the stochastic Fourier integral has been developed and continues to be used by Sabelfeld and coworkers [36, 42, 57, 72, 73]. They discretize the stochastic Fourier integral by a random choice of wavenumbers. This method is known as the Randomization Method or Double Randomization Method. The positive real axis in Fourier space is partitioned into $M$ successive, non-overlapping intervals $\left\{I_{j}\right\}_{j=1}^{M}$, usually chosen for purposes of Monte Carlo variance reduction [48, Sec. 6] so that the energies in each interval

$$
E_{j}=\int_{I_{j}} E(k) d k
$$


are equal.

Within each interval $I_{j}, \tilde{M}$ wavenumbers $\left\{k_{j}^{(m)}\right\}_{m=1}^{\tilde{M}}$ are chosen independently with a probability distribution weighted by the energy spectrum:

$$
\begin{aligned}
\operatorname{Prob}\left\{k_{j}^{(m)} \in A\right\} & =\int_{A} p_{j}(k) d k, \\
p_{j}(k) & = \begin{cases}\frac{E(k)}{E_{j}} & \text { for } k \in I_{j}, \\
0 & \text { for } k \notin I_{j} .\end{cases}
\end{aligned}
$$

The random wavenumbers so selected are then used as a basis for discretizing the Fourier stochastic integral representation (4):

$$
u_{\text {Rand }}(x)=\frac{1}{\sqrt{\tilde{M}}} \sum_{m=1}^{\tilde{M}} \sum_{j=1}^{M} \sqrt{2 E_{j}}\left[\xi_{j}^{(m)} \cos \left(2 \pi k_{j}^{(m)} x\right)+\eta_{j}^{(m)} \sin \left(2 \pi k_{j}^{(m)} x\right)\right],
$$

where $\left\{\xi_{j}^{(m)}, \eta_{j}^{(m)}\right\}_{j=1 \ldots M}$ are a collection of independent standard Gaussian random variables.

The velocity field simulated by the Randomization Method actually produces the correct correlation function (in the limit of large sample size) [48, 73]:

$$
\left\langle u_{\text {Rand }}(x+r) u_{\text {Rand }}(x)\right\rangle=\langle u(x+r) u(x)\rangle=R(r),
$$

which makes it a useful tool for simulating velocity fields with strong long range correlations. The drawback, however, is that $u_{\text {Rand }}(x)$ has non-Gaussian statistics not corresponding to any physical or ideal mathematical turbulence model $[13,73]$. (There is no real possibility of tuning the method to produce desired non-Gaussian features.) The departure from Gaussianity can be decreased by choosing larger values of $\tilde{M}$ by a central limit argument, because $u_{\text {Rand }}(x)$ can be thought of as an average of $\tilde{M}$ independently simulated velocity fields with one wavenumber chosen from each interval $I_{j}[38,48]$.

\subsection{Moving-Average Method}

Other types of Eulerian fluid Monte Carlo simulation methods can be derived from an alternative (and equivalent) physical-space representation of the Gaussian random field:

$$
u(x)=\int_{-\infty}^{\infty} G(x-r) d W(r)=\int_{-\infty}^{\infty} G(r) d W(x-r),
$$

where $d W(r)$ is a Gaussian white noise measure as defined in (5) which is convolved against the kernel

$$
G(x)=\int_{-\infty}^{\infty} \cos (2 \pi k x) E^{1 / 2}(|k|) d k .
$$

By a direct truncation and equispaced discretization of this physical space representation for the velocity field $v(x)$, we obtain the basis for the Moving Average simulation method first used by McCoy [55]:

$$
u_{\mathrm{MA}}(x)=\sum_{j=\lfloor x / \Delta r\rfloor-b}^{\lfloor x / \Delta r\rfloor+b} G\left(x-r_{j}\right) \xi_{j} \sqrt{\Delta r}
$$

Here $\Delta r$ denotes the width of the discretization, $b$ denotes the bandwidth of truncation of the stochastic convolution integral (6), $\lfloor x\rfloor$ denotes the greatest integer not exceeding $x,\left\{r_{j}=j \Delta r\right\}_{j=-\infty}^{\infty}$ 
are the equispaced grid points, and $\left\{\xi_{j}\right\}_{j=-\infty}^{\infty}$ is a collection of independent standard, real-valued Gaussian random variables. Note that one only needs to keep track of the finite set (which grows with time) of random variables $\xi_{j}$ associated to grid points which are within a bandwidth of the region which the particle(s) has explored. An efficient means of managing this kind of random number generation, which is important for practical success of the more elaborate Wavelet Method to be presented below, is described in [15].

One-sided variations of the moving average method have been used to simulate fractional Brownian motion $[22,50,51,52]$, though we do not recommend the use of the moving average method for simulating such random fields with strong long-range correlations due to its poor showing on test problems [14].

\subsection{Wavelet Method}

The physical space representation (6) of the random velocity field $u(x)$ can also be used as the basis for a hierarchical Monte Carlo which is particularly well suited, but not restricted, to simulating random fields with strong correlations extending over many length scales with or without the inertial-range scaling property (3). One proceeds by an exact expansion of the stochastic convolution integral (6) with respect to an orthonormal basis of wavelets $\left\{\phi_{m n}\right\}_{m, n=-\infty \ldots \infty}$ :

$$
\begin{aligned}
u(x) & =\sum_{m, n=-\infty}^{\infty} G \star \phi_{m n}(x) \xi_{m n}, \\
\phi_{m n}(x) & =2^{m / 2} \phi\left(2^{m} x-n\right) \quad m, n=0, \pm 1, \pm 2, \ldots,
\end{aligned}
$$

where $\left\{\xi_{m n}\right\}_{m, n=-\infty}^{\infty}$ is a collection of independent standard Gaussian random variables, and $\phi$ is a suitable Meyer wavelet [12]. The hierarchical structure of this representation is made clearer by writing

$$
\begin{aligned}
u(x) & =\sum_{m=-\infty}^{\infty} u_{m}\left(2^{m} x\right), \\
u_{m}(x) & =\sum_{n=-\infty}^{\infty} f_{m}(x-n) \xi_{m n}, \\
f_{m}(x) & =G_{m} \star \phi(x) \\
G_{m}(x) & =2^{-m / 2} G\left(2^{-m} x\right) .
\end{aligned}
$$

This exact expression must of course be truncated for a numerical implementation:

$$
\begin{aligned}
u_{\mathrm{FW}}(x) & =\sum_{m=0}^{M-1} u_{\mathrm{FW}, \mathrm{m}}\left(2^{m} x\right), \\
u_{\mathrm{FW}, \mathrm{m}}(x) & =\sum_{n=\lfloor x\rfloor-b}^{\lfloor x\rfloor+b} f_{\mathrm{FW}, m}(x-n) \xi_{m n} .
\end{aligned}
$$

The power of this wavelet method is that long-range correlations in the velocity field can be efficiently represented with reasonable values of $M$ and $b$ due to the hierarchical representation $[13$, 15]. It is crucial, however, to choose the Meyer wavelet $\phi$ so that the functions $f_{\mathrm{FW}, m}$ decay rapidly so that an accurate truncation of the sum over translates $n$ may be taken at moderate values of $b$. This can be accomplished by constructing the Fourier transform $\hat{\phi}$ of the Meyer wavelet using a $p$ th order perfect B-spline so that $\hat{\phi}$ is compactly supported away from the origin and has $p$ bounded 
derivatives. This will ensure that $f_{\mathrm{FW}, m}(x)$ decays at least as fast as $o\left(|x|^{-p}\right)$. Further theoretical and implementation details may be found in [13].

The wavelet Monte Carlo method described here is the one called the Fourier-Wavelet method in the literature $[13,48]$. This Fourier-Wavelet Method is more flexible than an earlier wavelet method [15], which also performed extraordinarly well but could only simulate velocity fields with an entirely self-similar scaling structure.

\subsection{Comparison of Eulerian fluid simulation methods}

The above Monte Carlo techniques have been tested by the examination of single particle diffusion and pair dispersion in synthetic shear flows generated by these methods $[14,15]$. The simulation results can be compared against exact analytical formulas for the statistics of the particle motion in ideal Gaussian random shear flows with specified correlation function [31]. Moreover, by taking different choices of correlation functions, the simulation techniques can be tested for a rich variety of statistical structures in the velocity field. Numerical studies of the capacity of the Randomization and Wavelet methods to generate velocity fields with a range of clean self-similar scaling have also been published $[13,15]$.

In these studies, the direct Fourier method is found seriously deficient due to its inherent periodicity artifact. For flows without strong long-range correlations, the Moving Average method gave good results but the Randomization method had difficulties for such flows with coherent oscillatory components (reflected in a negative tail to the correlation function). The Randomization method and Wavelet Methods appear to be the best candidates for simulations where the velocity field does have strong long-range correlations. The Wavelet Methods are particularly well-suited for simulating random fields with many decades of self-similar scaling, as amply demonstrated by the studies in $[13,15]$ and the clean validation of Richardson's law for pair dispersion in [17]. The Wavelet Methods, however, do have a rather high overhead cost, so the Randomization Method is actually more efficient when four or fewer decades of self-similar scaling behavior in the velocity field are desired. One must be cautious, however, with the non-Gaussian nature of the synthetic velocity field simulated by the Randomization Method [13]; the Wavelet Methods by contrast always simulate a Gaussian random field.

We emphasize that the above discussion concerns the ability of the Eulerian fluid simulation methods to simulate Gaussian random fields with prescribed correlation function or energy spectrum. The issue of the simulation of the non-Gaussian, intermittent features of turbulent flows is another matter $[42,72]$.

\section{Purely Lagrangian Simulation Methods}

Due to limited space, we endeavor here only to present some basic ideas behind the purely Lagrangian Monte Carlo methodology for turbulent systems, referring the reader to the references for details.

\subsection{Simulation of Fluid Motion and Properties}

We begin by considering a purely Lagrangian model for the dynamics of a turbulent fluid itself, in which the fluid evolution is described in terms of the motion of representative "fluid particles" rather than in terms of a nonlinear partial differential equation such as the Navier-Stokes equations. Models for the motion of immersed particles (which are generally of more applied interest) will be extended from the pure fluid Lagrangian model. 
The general idea is that the acceleration of fluid particles should rapidly decorrelate in time [61], so that the velocity $\mathbf{V}_{f}(t)$ of a fluid particle should evolve according to a continuous Markov process which may be expressed in general in terms of a stochastic differential equation (SDE) (2) [78]. By contrast, there can be long-range temporal correlations in the velocity field, particularly in high-Reynolds number turbulence, so a single stochastic differential equation for the position of a fluid particle $\mathbf{X}_{f}(t)$ would be a poor model.

One simple class of Lagrangian models for fluid particle motion has the velocity of a fluid particle evolve according to a diagonalized Langevin equation:

$$
\begin{aligned}
& d \mathbf{X}_{f}(t)=\mathbf{V}_{f}(t) d t \\
& d \mathbf{V}_{f}(t)=\left[-\mathbf{A}_{0}-\left(\mathbf{V}_{f}(t)-\overline{\mathbf{V}}_{\mathrm{f}}\right) / \tau_{f}\right] d t+\sigma d \mathbf{W}(t),
\end{aligned}
$$

where $\mathbf{A}_{0}$ is a mean acceleration, $\overline{\mathbf{V}}_{\mathrm{f}}$ is the mean fluid velocity, $\sigma$ reflects the amplitude of the unresolved random component of the fluid acceleration, and $\tau_{f}$ is the Lagrangian decorrelation time of a fluid particle [48, 77]. Such a model is too crude to capture the fine-scale features of fully developed turbulence, such as inertial-range scaling properties and intermittency, and will poorly describe the dynamics of particle pairs separated by distances within or below the inertial range of scales. However, the simple Langevin model (7) can be used as the basis for studies of turbulent systems in applications where one wishes to model some influence of turbulent mixing by the unresolved scales but is willing to neglect some of the statistical details of the mixing process.

The turbulence in such systems is almost always inhomogenous and changing in time, so one should naturally allow the parameters $\mathbf{A}_{0}, \tau_{f}, \overline{\mathbf{V}}_{\mathrm{f}}$, and $\sigma$ to depend on the local mean properties of the fluid at the current position $\mathbf{X}_{f}(t)$ of the fluid particle. Moreover, for anisotropic turbulence, the drag term is often written as a tensor multiplied by the velocity difference $\mathbf{V}_{f}(t)-\overline{\mathbf{V}}_{\mathrm{f}}$ [67]. For applications in modeling transport of immersed particles where the one-point velocity statistics are time-invariant and can be taken to known a priori (perhaps through field observations of an atmospheric or oceanic region ), then one can define appropriate deterministic functions of position $\mathbf{A}_{0}(\mathbf{x}), \overline{\mathbf{V}}_{\mathrm{f}}(\mathbf{x}) \tau_{f}(\mathbf{x})$, and $\sigma(\mathbf{x})$ for the mean acceleration, mean velocity, Lagrangian correlation time, and root mean square turbulent velocity fluctuation, and set the parameters appearing in (7) to be the evaluation of these deterministic functions at the current position $\mathbf{x}=\mathbf{X}_{f}(t)$ of the fluid particle [79].

When such a priori information is not available, particularly if the modeling interest is on determining the statistics of the turbulent fluid itself, one must instead compute the mean local fluid properties as the simulation proceeds. The natural way to do this is to define the mean of a fluid property at a given location in terms of an ensemble average over the properties of fluid particles within a certain neighborhood of that location $[60,68,84,86]$. The mean velocity $\overline{\mathbf{V}}_{\mathrm{f}}(\mathbf{x}, t)$ can be computed directly in this fashion. The Lagrangian correlation time $\tau_{f}(\mathbf{x}, t)$ is modeled similarly by associating an instantaneous local turbulent frequency $\Omega(t)$ to each fluid particle, evolving $\Omega(t)$ according to an additional SDE, and then taking a local ensemble average of the particle values $\Omega^{-1}(t)$ to obtain a local value for the Lagrangian correlation time $\tau_{f}(\mathbf{x}, t)[60,66]$. The mean acceleration $\mathbf{A}_{0}(\mathbf{x}, t)$ is usually modeled in terms of a pressure gradient $[59,67]$ which must be computed by more elaborate means (enforcing a form of incompressibility [60] or linking it by an equation of state to the local mean density in a compressible flow [84]). The magnitude of the turbulent accelerations $\sigma(\mathbf{x}, t)$ is often related to the local energy dissipation rate through an appeal to Kolmogorov's theory $[67,65]$. We remark that the frictional damping time $\tau_{f}(\mathbf{x}, t)$ and the noise amplitude $\sigma(\mathbf{x}, t)$ need not obey the fluctuation-dissipation relations of statistical mechanics when the turbulent system is not in a statistically stationary state $[59,65,67]$ 


\subsection{Simulation of Immersed Particles}

The motion of immersed solid particles can be modelled in a similar fashion. One approach for dense particles is to relate the dynamics of the position $\mathbf{X}_{p}(t)$ and velocity $\mathbf{V}_{p}(t)$ of an immersed particle to the fluid velocity $\mathbf{V}_{s}(t)$ "seen" by the particle [70]:

$$
\begin{aligned}
& d \mathbf{X}_{p}(t)=\mathbf{V}_{p}(t) d t \\
& d \mathbf{V}_{p}(t)=\left(\mathbf{V}_{s}(t)-\mathbf{V}_{p}(t)\right) / \tau_{p} d t+\mathbf{A}_{p}(t) d t,
\end{aligned}
$$

where $\tau_{p}$ is a particle momentum relaxation time scale related to its inertia and drag, and $\mathbf{A}_{p}(t)$ represents acceleration due to external forces (such as gravity) acting on the immersed particle. The momentum relaxation time $\tau_{p}$ can be taken as constant for homogenous, low Reynolds number flows, but generally depends on the location and velocity of the particle $[69,70]$. The fluid velocity seen by the immersed particle, $\mathbf{V}_{s}(t)$, is modeled by an equation of the form (7), but with the Lagrangian correlation time generally decreased from that of a pure fluid particle model because the immersed particle "sees" different fluid particles as it moves around [70]. A more sophisticated model for immersed particle motion in homogenous turbulent flows is offered in [69], but this approach does not yet seem to have been extended to inhomogenous turbulent flows. A more detailed exposition of the purely Lagrangian modeling of the motion of particles immersed in a turbulent fluid can be found in the paper by Minier [58] in this volume.

\subsection{Hybrid LES/Monte Carlo Schemes}

One particularly interesting use to which the purely Lagrangian Monte Carlo simulation methodology has been put is as a subgrid scale model for turbulent combustion studies [33, 62]. In these simulations, the mean hydrodynamic variables are updated according to a large eddy simulation (LES) scheme (based on the Navier-Stokes PDE), while the combustion and mixing of the chemical reactants is handled by a purely Lagrangian Monte Carlo model. That is, the chemical reactants are represented as a collection of particles, which move about according to certain stochastic differential equations and also change their chemical composition due to mixing and reaction as they move. Note that the representative particles in such as a scheme should not be thought of as an actual chemical entity; they are rather representative parcels containing some mixture of the chemicals present in the fluid. The coefficients of the stochastic differential equations governing motion and chemical mixing are obtained from local mean fluid properties produced by the LES, and the LES equations contain terms involving local averages over the chemical state of nearby Lagrangian particles [33]. The incorporation of the Monte Carlo element into the turbulent combustion simulation has been shown to significantly improve the accuracy of the results as compared to a pure LES simulation [33,62]. The reason is that the Monte Carlo component incorporates some effects and dynamics of small-scale fluctuations in the chemical reactant density which are not otherwise incorporated in the LES.

\subsection{Theoretical Modeling Issues}

In formulating Lagrangian models, one must pay heed not only to making physically motivated choices of the parameters and functions in the model, but also to ensuring that grossly unphysical artifacts are not generated. A variety of Langevin models of the form (7) have been shown to violate seriously the very natural well-mixed condition [78] when applied to inhomogenous turbulence [44]. The well-mixed condition is the physically natural property that if the immersed particle density equals the fluid density at a given point in time, it will continue to do so forever. In other words, the simulation scheme should not "unmix" a well-mixed suspension of immersed particles in a 
fluid. A Langevin model of the form (7) can be made to obey the well-mixed condition if the mean acceleration $\mathbf{A}_{0}$ is properly computed in terms of an averaged local pressure gradient [65], as in the model presented in [29,67]. Other purely Lagrangian models of the more general form (2) which do respect the well-mixed condition have been formulated [42, 72, 78], but they are considerably more complex and not often used in practical applications.

Another way in which the simple model (7) can be improved is to introduce coupling between different particles which better reflect some of the small-scale statistical features of turbulence [ 42 , $72]$.

\section{Conclusion}

In this introductory review, we have briefly described a few Monte Carlo approaches to the simulation of turbulent systems. The Eulerian fluid simulation approaches are useful primarily for theoretical studies of turbulent diffusion and combustion, because well-designed methods (such as the Randomization or Wavelet-based Methods [13]) can generate very good approximations to a certain class of well-defined random flow models. By defining these random flows to have certain features (such as an inertial scaling range and certain geometric properties), one can study in a well-controlled way how immersed particles and/or chemical reactants respond to these features in the model $[2,5,17,41,48]$.

The purely Lagrangian Monte Carlo approach is, on the other hand, much less expensive than the Eulerian fluid simulation approach and can be adapted to inhomogenous turbulent systems prevalent in applications. There is still much to understand, however, in the design of good purely Lagrangian models. Both theoretical criteria $[42,44,72,65,78]$ and practical implementation issues $[68,86]$ should continue to inform the development of these promising simulation tools.

\section{Acknowledgements}

The author would like to acknowledge the anonymous referee for some helpful comments which clarified the discussion of Lagrangian models.

\section{References}

[1] J. Bonet Avalos and A. D. Mackie. Dissipative particle dynamics with energy conservation. Europhys. Lett., 40(2):141-146, 1997.

[2] Marco Avellaneda, Jr. Elliott, Frank, and Christopher Apelian. Trapping, percolation, and anomalous diffusion of particles in a two-dimensional random field. J. Statist. Phys., 72(56):1227-1304, 1993 .

[3] Marco Avellaneda and Andrew J. Majda. Mathematical models with exact renormalization for turbulent transport. Comm. Pure Appl. Math., 131:381-429, 1990.

[4] Marco Avellaneda and Andrew J. Majda. Mathematical models with exact renormalization for turbulent transport, II: Fractal interfaces, non-Gaussian statistics and the sweeping effect. Comm. Pure Appl. Math., 146:139-204, 1992.

[5] Marco Avellaneda, S. Torquato, and I. C. Kim. Diffusion and geometric effects in passive advection by random arrays of vortices. Phys. Fluids A, 3(8):1880-1891, August 1991. 
[6] Peter Biechele, Heinz-Peter Breuer, and Francesco Petruccione. Non-equilibrium Monte Carlo simulation of decaying Navier-Stokes turbulence. Phys. Lett. A, 256:147-152, May 311999.

[7] A. Bourlioux and A. J. Majda. An elementary model for the validation of flamelet approximations in non-premixed turbulent combustion. Combust. Theory Model., 4(2):189-210, 2000.

[8] H. P. Breuer and F. Petruccione. Mesoscopic modelling and stochastic simulations of turbulent flows. In Nonlinear stochastic PDEs (Minneapolis, MN, 1994), pages 261-291. Springer, New York, 1996.

[9] Renè A. Carmona, Stanislav A. Grishin, and Stanislav A. Molchanov. Massively parallel simulations of motions in a Gaussian velocity field. In Stochastic modelling in physical oceanography, volume 39 of Progr. Prob., pages 47-68. Birkhäuser Boston, Boston, 1996.

[10] Alexandre J. Chorin. Vorticity and turbulence. Number 103 in Applied Mathematical Sciences. Springer-Verlag, New York, 1994.

[11] G. T. Csanady. Turbulent diffusion in the environment, volume 3 of Geophysics and Astrophysics Monographs. D. Reidel, Dordrecht, Boston, Lancaster, Tokyo, 1973.

[12] Ingrid Daubechies. Ten lectures on wavelets, volume 61 of CBMS-NSF Regional Conference Series in Applied Mathematics. Society for Industrial and Applied Mathematics (SIAM), Philadelphia, PA, 1992.

[13] Frank W. Elliott, Jr, David J. Horntrop, and Andrew J. Majda. A Fourier-wavelet Monte Carlo method for fractal random fields. J. Comp. Phys, 132(2):384-408, 1997.

[14] Frank W. Elliott, Jr, David J. Horntrop, and Andrew J. Majda. Monte Carlo methods for turbulent tracers with long range and fractal random velocity fields. Chaos, 7(1):39-48, 1997.

[15] Frank W. Elliott, Jr and Andrew J. Majda. A wavelet Monte Carlo method for turbulent diffusion with many spatial scales. J. Comp. Phys, 113(1):82-111, July 1994.

[16] Frank W. Elliott, Jr and Andrew J. Majda. A new algorithm with plane waves and wavelets for random velocity fields with many spatial scales. J. Comp. Phys, 117:146-162, 1995.

[17] Frank W. Elliott, Jr and Andrew J. Majda. Pair dispersion over an inertial range spanning many decades. Phys. Fluids, 8(4):1052-1060, 1996.

[18] Frank W. Elliott, Jr, Andrew J. Majda, David J. Horntrop, and Richard M. McLaughlin. Hierarchical Monte Carlo methods for fractal random fields. J. Statist. Phys., 81:717, 1995.

[19] P. Español. Dissipative particle dynamics with energy conservation. Europhys. Lett., 40(6):631-636, 1997.

[20] P. Español and P. Warren. Statistical mechanics of dissipative particle dynamics. Europhys. Lett., 30(4):191-196, May 11995.

[21] Pep Español. Fluid particle model. Phys. Rev. E, 57(3):2930-2948, March 1998.

[22] Jens Feder. Fractals, chapter 9-14, pages 163-243. Physics of Solids and Liquids. Plenum Press, New York and London, 1988.

[23] U. Frisch, A. Mazzino, A. Noullez, and M. Vergassola. Lagrangian method for multiple correlations in passive scalar advection. Phys. Fluids, 11(8):2178-2186, 1999. The International Conference on Turbulence (Los Alamos, NM, 1998). 
[24] U. Frisch, A. Mazzino, and M. Vergassola. Intermittency in passive scalar advection. Phys. Rev. Lett., 80(25):5532-5535, June 221998.

[25] Uriel Frisch. Turbulence. Cambridge University Press, Cambridge, 1995. The legacy of A. N. Kolmogorov.

[26] Omri Gat and Reuven Zeitak. Multiscaling in passive scalar advection as stochastic shape dynamics. Phys. Rev. E, 57(5):5511-5519, May 1998.

[27] Robert D. Groot and Patrick B. Warren. Dissipative particle dynamics: Bridging the gap between atomistic and mesoscopic simulation. J. Chem. Phys., 107(11):4423-4435, September 15 1997.

[28] D. B. Haidvogel. On the feasibility of particle tracking in Eulerian ocean models. Ocean Model., 45:4-9, 1982.

[29] D. C. Haworth and S. B. Pope. A pdf modeling study of self-similar turbulent free shear flows. Phys. Fluids, 30:1026, 1987.

[30] P. J. Hoogerbrugge and J. M. V. A. Koelman. Simulating microscopic hydrodynamic phenomena with dissipative particle dynamics. Europhys. Lett., 19(3):155-160, June 11992.

[31] D. Horntrop and A. Majda. An overview of Monte Carlo simulation techniques for the generation of random fields. In P. Muller and D. Henderson, editors, Monte Carlo Simulations in Oceanography, pages 67-79, 1997. Proceedings of the Ninth 'Aha Huliko'a Hawaiian Winter Workshop.

[32] David J. Horntrop and Andrew J. Majda. Subtle statistical behavior in simple models for random advection-diffusion. J. Math. Sci. Univ. Tokyo, 1:1-48, 1994.

[33] F. A. Jaberi, P. J. Colucci, S. James, P. Givi, and S. B. Pope. Filtered mass density function for large-eddy simulation of turbulent reacting flows. J. Fluid Mech., 401:85-121, 1999.

[34] A. R. Kerstein. Linear-eddy modeling of turbulent transport. 4. Structure of diffusion flames. Comb. Sci. and Tech., 81(1-3):75-96, 1992.

[35] Alan R. Kerstein. One-dimensional turbulence: model formulation and application to homogeneous turbulence, shear flows, and buoyant stratified flows. J. Fluid Mech., 392:277-334, 1999.

[36] A. Kolodko, K. Sabelfeld, and W. Wagner. A stochastic method for solving Smoluchowski's coagulation equation. Math. Comput. Simulation, 49(1-2):57-79, 1999.

[37] Robert H. Kraichnan. Small-scale structure of a scalar field convected by turbulence. Phys. Fluids, 11(5):945-953, May 1968.

[38] Robert H. Kraichnan. Diffusion by a random velocity field. Phys. Fluids, 13(1):22-31, January 1970.

[39] Robert H. Kraichnan. Anomalous scaling of a randomly advected passive scalar. Phys. Rev. Lett., 72(7):1016-1019, February 1994.

[40] Steven K. Krueger. Linear eddy modeling of entrainment and mixing in stratus clouds. J. of Atmospheric Science, 50(18):3078-3090, 1993. 
[41] O. A. Kurbanmuradov. Stochastic Lagrangian models for two-particle relative dispersion in high-Reynolds number turbulence. Monte Carlo Methods Appl., 3(1):37-52, 1997.

[42] O. A. Kurbanmuradov and K. K. Sabelfeld. Statistical modelling of turbulent motion of particles in random velocity fields. Sov. J. Numer. Anal. Math. Modelling, 4(1):53-68, 1989.

[43] Marcel Lesieur. Turbulence in fluids. Number 1 in Fluid Mechanics and its Applications. Kluwer, Dordrecht, second revised edition, 1990.

[44] J. M. MacInnes and F. V. Bracco. Stochastic particle dispersion modeling and the tracerparticle limit. Phys. Fluids A, 4(12):2809-2824, December 1992.

[45] Andrew Majda and Panagiotis Souganidis. The effect of turbulence on mixing in prototype reaction-diffusion systems. Comm. Pure Appl. Math., 53(10):1284-1304, 2000.

[46] Andrew J. Majda. Random shearing direction models for isotropic turbulent diffusion. $J$. Statist. Phys., 25(5/6):1153-1165, 1994.

[47] Andrew J. Majda. Real world turbulence and modern applied mathematics. In Mathematics: frontiers and perspectives, pages 137-151. Amer. Math. Soc., Providence, RI, 2000.

[48] Andrew J. Majda and Peter R. Kramer. Simplified models for turbulent diffusion: Theory, numerical modelling and physical phenomena. Phys. Rep., 314(4-5):237-574, June 1999.

[49] Benoit B. Mandelbrot. The fractal geometry of nature. W.H. Freeman, San Francisco, New York, updated and augmented edition, 1983.

[50] Benoit B. Mandelbrot and James R. Wallis. Computer experiments with fractional Gaussian noises. Part 1, Averages and variances. Water Resour. Res., 5(1):228-241, February 1969.

[51] Benoit B. Mandelbrot and James R. Wallis. Computer experiments with fractional Gaussian noises. Part 2, Rescaled ranges and spectra. Water Resour. Res., 5(1):242-259, February 1969.

[52] Benoit B. Mandelbrot and James R. Wallis. Computer experiments with fractional Gaussian noises. Part 3, Mathematical appendix. Water Resour. Res., 5(1):260-267, February 1969.

[53] Martin R. Maxey. Equation of motion for a small rigid sphere in a nonuniform flow. Phys. Fluids, 26(4):883-889, April 1983.

[54] W. D. McComb. The physics of fluid turbulence, volume 25 of Oxford Engineering Science Series. Clarendon Press, New York, 1991.

[55] A. McCoy. PhD thesis, University of California at Berkeley, 1975. Department of Mathematics.

[56] S. Menon and W. H. Calhoon, Jr. Subgrid mixing and molecular transport modeling in a reacting shear layer. In 26th Symp. (Intl.) on Combustion, pages 59-66. The Combustion Institute, 1996.

[57] Gennadii A. Mikhailov. Optimization of weighted Monte Carlo methods, chapter 6, pages 152-156, 161-164. Springer Series in Computational Physics. Springer-Verlag, Berlin, 1992.

[58] Jean-Pierre Minier, 2000. Present volume.

[59] Jean-Pierre Minier and Jacek Pozorski. Derivation of a PDF model for turbulent flows based on principles from statistical physics. Phys. Fluids, 9(6):1748-1753, 1997. 
[60] Jean-Pierre Minier and Jacek Pozorski. Wall-boundary conditions in probability density function methods and application to a turbulent channel flow. Phys. Fluids, 11(9):2632-2644, September 1999.

[61] A. S. Monin and A. M. Yaglom. Statistical fluid mechanics: mechanics of turbulence, volume 2, page 370. MIT Press, Cambridge, MA, 1975.

[62] A. Obieglo, J. Gass, and D. Poulikakos. Comparative study of modeling a hydrogen nonpremixed turbulent flame. Combust. Flame, 122:176-194, 2000.

[63] I. Pagonabarraga, M. H. J. Hagen, and D. Frenkel. Self-consistent dissipative particle dynamics algorithm. Europhys. Lett., 42(4):377-382, 1998.

[64] S. B. Pope. The probability approach to the modelling of turbulent reacting flows. Combust. Flame, 27:299-312, 1976.

[65] S. B. Pope. Consistency conditions for random-walk models of turbulent dispersion. Phys. Fluids, 30(8):2374-2379, August 1987.

[66] S. B. Pope. Application of the velocity-dissipation probability density function model to inhomogenous turbulent flows. Phys. Fluids A, 3:1947, 1991.

[67] S. B. Pope. Lagrangian PDF methods for turbulent flows. In Annual review of fluid mechanics, Vol. 26, pages 23-63. Annual Reviews, Palo Alto, CA, 1994.

[68] S. B. Pope. Particle method for turbulent flows: Integration of stochastic model equations. J. Comp. Phys, 117:332-349, 1995.

[69] Jacek Pozorski and Jean-Pierre Minier. On the Lagrangian turbulent dispersion models based on the Langevin equation. International Journal of Multiphase Flow, 24:913-945, 1998.

[70] Jacek Pozorski and Jean-Pierre Minier. Probability density function modeling of dispersed two-phase turbulent flows. Phys. Rev. E, 59(1):855, January 1999.

[71] K. K. Sabelfeld, 2000. Present volume.

[72] K. K. Sabelfeld and O. Kurbanmuradov. Two-particle stochastic Eulerian-Lagrangian models of turbulent dispersion. Math. Comput. Simulation, 47(2-5):429-440, 1998. IMACS Seminar on Monte Carlo Methods (Brussels, 1997).

[73] Karl K. Sabelfeld. Monte Carlo methods in boundary value problems, chapter 1,5, pages 3147,228-238. Springer Series in Computational Physics. Springer-Verlag, Berlin, 1991.

[74] Katepalli R. Sreenivasan. Fluid turbulence. Rev. Mod. Phys., 71(2):S383-S395, 1999.

[75] Katepalli R. Sreenivasan and R. A. Antonia. The phenomenology of small-scale turbulence. In Annual review of fluid mechanics, Vol. 29, volume 29 of Annu. Rev. Fluid Mech., pages 435-472. Annual Reviews, Palo Alto, CA, 1997.

[76] P. Tanga and A. Provenzale. Dynamics of advected tracers with varying buoyancy. Physica D, 76(1-3):202-215, September 11994.

[77] H. Tennekes and J. L. Lumley. A first course in turbulence. MIT Press, Cambridge, MA, 1972. 
[78] D. J. Thomson. Criteria for the selection of stochastic models of particle trajectories in turbulent flows. J. Fluid Mech., 180:529-556, 1987.

[79] H. van Dop, F. T. M. Nieuwstadt, and J. C. R. Hunt. Random walk models for particle displacements in inhomogenous unsteady turbulent flows. Phys. Fluids, 28(6):1639-1653, June 1985.

[80] P. R. Van Slooten, Jayesh, and S. B. Pope. Advances in PDF modeling for inhomogeneous turbulent flows. Phys. Fluids, 10(1):246-265, 1998.

[81] M. Vergassola, 2000. Present volume.

[82] J. A. Viecelli and E. H. Canfield, Jr. Functional representation of power-law random fields and time series. J. Comp. Phys, 95:29-39, 1991.

[83] Richard F. Voss. Random fractal forgeries. In Rae A. Earnshaw, editor, Fundamental algorithms for computer graphics, volume 17 of NATO ASI Series F: Computer and System Sciences, pages 805-835, Berlin, 1985. NATO Science Affairs Divison, Springer-Verlag.

[84] Walter C. Welton. Two-dimensional PDF/SPH simulations of compressible turbulent flows. J. Comput. Phys., 139(2):410-443, 1998.

[85] Forman A. Williams. Combustion theory: the fundamental theory of chemically reacting flow systems, chapter 3,7. Addison-Wesley Series in Engineering Science. Addison-Wesley, Reading, MA, USA, 1965.

[86] J. Xu and S. B. Pope. Assessment of numerical accuracy of PDF/Monte Carlo methods for turbulent reacting flows. J. Comp. Phys, 152:192-230, 1999.

[87] A. M. Yaglom. Correlation theory of stationary and related random functions. Volume I: Basic results. Springer-Verlag, Berlin, 1987.

[88] N. Zouari and A. Babiano. Experiences numériques lagrangiennes à partir de modéles eulériens. Atmosphere Ocean, 28:345-364, 1990. 